\title{
Ressignificando o pensamento computacional na perspectiva inclusiva
}

\author{
Resignifying computational thinking from an inclusive perspective \\ Resignificar el pensamiento computacional desde una perspectiva inclusiva
}

Claudiane Figueiredo Ribeiro ORCID: https://orcid.org/0000-0003-1130-0036 Universidade Federal Fluminense, Brasil E-mail: claudianefr@id.uff.br

Luciana da Silva Goudinho ORCID: https://orcid.org/0000-0003-3925-3366 Universidade Federal Fluminense, Brasil

E-mail: lucianagoudinho@id.uff.br

Sandro Miranda de Rezende ORCID: https://orcid.org/0000-0002-1179-3443 Universidade Federal Fluminense, Brasil

E-mail: sandromiranda@id.uff.br

Ruth Maria Mariani Braz

ORCID: https://orcid.org/0000-0003-2224-9643 Universidade Federal Fluminense, Brasil

E-mail: ruthmariani@yahoo.com.br

Roberta Carvalho de Souza

ORCID: https://orcid.org/0000-0002-3831-5564 Universidade Federal Fluminense, Brasil E-mail: souzaroberta@id.uff.br

Maria Cristina Barbosa Mendes ORCID: https://orcid.org/0000-0001-6924-3893

Universidade Federal de Juiz de Fora, Brasil

E-mail: mariacristinabarbosamendes@gmail.com

Sandra Mello de Menezes Felix de Souza

ORCID: https://orcid.org/0000-0003-2999-7708

Universidade Federal Fluminense, Brasil E-mail: sandramello1907@gmail.com

Ilma Rodrigues de Souza Fausto

ORCID: https://orcid.org/0000-0003-3850-5066 Instituto Federal de Educação Ciência e Tecnologia de Rondônia, Brasil E-mail: Ilma.rodrigues@ifro.edu.br Elaine Alves Leite

ORCID: https://orcid.org/0000-0002-7020-4530 Universidade Federal Fluminense, Brasil E-mail: elaineleite@id.uff.br

João Henrique Lopes Spies ORCID: https://orcid.org/0000-0002-7410-8490 Universidade Federal Fluminense, Brasil E-mail: jhspies@id.uff.br

Alessandra Furtado de Oliveira ORCID: https://orcid.org/0000-0001-5696-7606 Universidade Federal Fluminense, Brasil

E-mail: afurtadodeoliveiranovaes@yahoo.com.br

Sandro Medeiros Portella

ORCID: http://orcid.org/0000-0002-8823-0032 Instituto Nacional de Educação de Surdos, Brasil sandro_portella@hotmail.com.br

Michele Joia da Silva

ORCID: https://orcid.org/0000-0001-6033-9290 Universidade Federal Fluminense, Brasil E-mail: michelejoia92@gmail.com

Mírian Renata Medeiros dos Santos Valei ORCID: https://orcid.org/0000-0003-0988-5872 Universidade Federal Fluminense, Brasil E-mail: mirianrenata06@gmail.com Sérgio Crespo Coelho da Silva Pinto ORCID: http://orcid.org/0000-0001-6914-2398

Universidade Federal Fluminense, Brasi E-mail: screspo@id.uff.br 


\section{Resumo}

O pensamento computacional engloba habilidades necessárias para se trabalhar a resolução de problemas e, no campo educacional, abrange discussões sobre como seria possível aprimorarmos práticas pedagógicas relacionadas à inclusão de pessoas com deficiência e altas habilidades ou superdotação. Esta é uma pesquisa com relatos de experiências do grupo de pesquisa "Tecnologias Computacionais no ensino e aprendizagem na ótica da Diversidade, Inclusão e Inovação", reunidos no período da pandemia. Foi realizado o levantamento dos artigos existentes nas bases de dados Web of Science e Google Acadêmico para discussão de propostas relacionadas a novas formas de pensar em metodologias de ensino, envolvendo o pensamento computacional e a inclusão. Como resultado propomos novos rumos de análise que favoreçam práticas pedagógicas inclusivas, e apontamos para a reformulação dos pilares do pensamento computacional, a partir de necessidades específicas de ensino, sugerindo um passo a passo de trabalho para todos aqueles interessados em fomentar uma educação efetivamente mais inclusiva. Concluímos, então, que o pensamento computacional inclusivo é aplicável na educação formal ou informal e, é necessário para o desenvolvimento holístico dos sujeitos, diante das demandas do século XXI.

Palavras-chave: Pensamento computacional; Inclusão; Tecnologia da informação e comunicação; Pessoas com deficiências; Altas habilidades ou superdotação.
\end{abstract}

\begin{abstract}
Computational thinking includes skills needed to work with problem solving and, in the educational field, it covers discussions about how it would be possible to improve pedagogical practices related to the inclusion of people with disabilities and high abilities or giftedness. This research reports experiences of the research group "Computing Technologies in teaching and learning from the perspective of Diversity, Inclusion, and Innovation". A survey of existing articles in the Web of Science and Google Academic databases was carried out to discuss proposals related to new ways of thinking about teaching methodologies, involving computational thinking and inclusion. As a result, we propose new approaches that favor inclusive pedagogical practices, we point to the reformulation of the pillars of computational thinking, based on specific teaching needs, suggesting a step-by-step work for all those interested in fostering an education that is effectively more inclusive. We conclude, then, that inclusive computational thinking is applicable in formal or informal education, and is necessary for the holistic development of subjects, facing the demands of the 21 st century.
\end{abstract}

Keywords: Computational thinking; Inclusion; Information and communication technology; People with disabilities; High abilities or giftedness.

\title{
Resumen
}

El pensamiento computacional engloba las habilidades necesarias para trabajar en la resolución de problemas y, en el ámbito educativo, abarca los debates sobre cómo sería posible mejorar las prácticas pedagógicas relacionadas con la inclusión de personas con discapacidades y altas capacidades o superdotadas. Se trata de una investigación con informes de experiencias del grupo de investigación "Tecnologías informáticas en la enseñanza y el aprendizaje desde la perspectiva de la diversidad, la inclusión y la innovación". Se realizó un relevamiento de los artículos existentes en las bases de datos Web of Science y Google Académico para discutir las propuestas relacionadas con las nuevas formas de pensar en las metodologías de enseñanza, involucrando el pensamiento computacional y la inclusión. Como resultado, proponemos nuevas formas de análisis que favorezcan las prácticas pedagógicas inclusivas, e apuntamos a la reformulación de los pilares del pensamiento computacional, a partir de las necesidades específicas de la enseñanza, sugiriendo un trabajo paso a paso para todos los interesados en fomentar una educación efectivamente más inclusiva. Concluimos, entonces, que el pensamiento computacional inclusivo es aplicable en la educación formal o informal, y es necesario para el desarrollo holístico de los sujetos, considerando las demandas del siglo XXI.

Palabras clave: Pensamiento computacional; Inclusión; Tecnologías de la información y la comunicación; Personas con discapacidad; Altas capacidades o superdotación.

\section{Introdução}

O fenômeno da globalização mostra que, cada vez mais cedo, os indivíduos têm acesso aos meios das tecnologias digitais de informação e comunicação (TDIC), antes mesmo de completarem dois anos de idade. Desde tenra idade, portanto, estão em contato diário com celulares, televisões e até mesmo aos computadores.

As TDIC são ferramentas que possibilitam ao ser humano expressar o seu pensamento: proporcionam o desenvolvimento cultural e educacional de forma solidária e dinâmica, auxiliam na partilha de informações e contribuem na formação de cidadãos críticos. Por isso, essas ferramentas vêm sendo cada dia mais utilizadas no segmento educacional, permitindo assim o desenvolvimento e crescimento da educação do país. 
Nesse contexto, o Pensamento Computacional (PC) é uma forma de uso dessas tecnologias, desenvolvidas para facilitar a vida das pessoas na produção de conhecimentos, em espaços diversificados e com o fim de estabelecer relações de como pensar, agir, aprender e se comunicar. Com o pensamento computacional procuramos alternativas didáticas, seja através de atividades desplugadas ou plugadas, trabalhando na diversidade para a inclusão de educandos, pautados, por exemplo, em "Piaget [que] afirma que é necessário que o sujeito interaja com os objetos, pois a partir disso surgiram as dúvidas e incertezas responsáveis pelo desequilíbrio, que irá desencadear uma série de processos mentais que levarão à construção do conhecimento" (como citado em Pinto \& Nascimento, 2018, p.309).

De acordo com a Lei Brasileira de Inclusão (Lei no 13.146, de 6 de julho de 2015):

Art. $2^{\circ}$ Considera-se pessoa com deficiência aquela que tem impedimento de longo prazo de natureza física, mental, intelectual ou sensorial, o qual, em interação com uma ou mais barreiras, pode obstruir sua participação plena e efetiva na sociedade em igualdade de condições com as demais pessoas (Brasil, 2015, p.1).

A educação inclusiva, assim como a educação especial, faz uso de tecnologias para facilitar algumas ações cotidianas que visam a minimizar as barreiras encontradas pelas pessoas com deficiência, de modo a facilitar sua participação e interação com a sociedade. Diferentes pessoas têm diferentes maneiras de acionar ou realizar os quatro pilares do pensamento computacional em suas tarefas escolares ou do dia-a-dia, sendo eles: abstrair, decompor um problema, realizar uma padronização e usar os algoritmos. Desta forma algumas questões se fazem necessárias para reflexão: (1) Como trabalhar as habilidades do Pensamento Computacional para um público com algum tipo de deficiência? (2) Como trabalhar uma arquitetura pedagógica inclusiva para potencializar as habilidades do Pensamento Computacional? (3) Como ressignificar os conceitos do Pensamento Computacional para pessoas com deficiência?

$\mathrm{O}$ aluno estimulado a partir das bases do pensamento computacional pode desenvolver diferentes competências, tais como a capacidade de análise, interpretação, compreensão de pontos relevantes de uma questão, além de permitir a conexão de diversos conhecimentos e habilidades. Assim podemos trabalhar de forma diferenciada, de acordo com as necessidades dos alunos, através da oferta de uma abordagem que utilize o pensamento computacional para resolver situações problemas.

A combinação do pensamento crítico com os fundamentos da computação define uma metodologia para resolver problemas, denominada Pensamento Computacional (Wing, 2006). Esta visão defende que a cultura do computador ajuda a sociedade não somente a aprender, mas especialmente oferece uma nova maneira de aprender a aprender (Lu \& Fletcher, 2009; Papert, 1980). Para Wing (2006), o pensamento computacional desenvolve a capacidade analítica e investigativa para chegar a uma decisão a respeito de situações que envolvam a natureza, a sociedade, a ciência e a tecnologia; a capacidade de comunicação para ouvir, interpretar e expressar diferentes pontos de vista; a imaginação para colocar-se no lugar do outro, compreendendo concepções, argumentos e pontos de vista diferentes dos seus com sensibilidade e sem preconceitos.

Primeiramente, fazemos alusão às políticas públicas das TIC vigentes e as competências gerais a serem desenvolvidas de acordo com a BNCC (Brasil, 2018) sobre o uso do Pensamento Computacional. É levantada, então, uma discussão sobre o Pensamento Computacional e os seus pilares aplicáveis à educação de todos, de forma plugada ou desplugada, visando aqueles que mais precisam de um trabalho diferenciado, seja através de jogos, atividades manuais ou cognitivas nos seus diversos níveis de complexidade e público. Por fim, com o objetivo de divulgar as discussões, estudos e experiências acerca do Pensamento Computacional Inclusivo do grupo de pesquisa do Conselho Nacional de Desenvolvimento Científico e Tecnológico (CNPq), intitulado Tecnologias Computacionais no ensino e aprendizagem na ótica da Diversidade, Inclusão e Inovação (TeCEADI+), compomos um arcabouço teórico-tecnológico sobre o pensamento computacional inclusivo para atender as demandas educacionais de pessoas com deficiência e altas habilidade ou superdotação. 


\subsection{Fundamentação teórica}

Historicamente, percebe-se que o processo de inclusão das pessoas com deficiência nas escolas regulares perpassou diversos momentos de conflitos, tendo como bases situações de rejeição, preconceito e discriminação, uma vez que, esses sujeitos eram considerados incapazes de aprender, dessa maneira foram colocados à margem da sociedade ao longo dos séculos. No Brasil as mudanças nas políticas públicas educacionais direcionadas para essas pessoas só ocorreram a partir do século XX (Giroto, Poker \& Omote, 2012).

Nesse contexto, a Política Nacional de Educação Especial na Perspectiva da Educação Inclusiva do Ministério da Educação e Cultura (MEC), recomenda a implantação de políticas públicas para que alunos com deficiência, transtornos globais do desenvolvimento e altas habilidades ou superdotação tenham garantido o acesso, a participação e o aprendizado nas escolas comuns (Brasil, 2008). Dessa maneira: "Os sistemas educacionais precisam atender às necessidades de todos os estudantes" (Unesco, 2020, p. 22).

Ainda, o Ministério da Educação e Cultura (MEC), através do Conselho Nacional de Educação/Câmara de Educação Básica, vem possibilitando uma discussão na sociedade civil sobre as normas da Computação na Educação Básica - de acordo com a Base Nacional Comum Curricular-BNCC (Brasil, 2018). Esse documento menciona que, na década de 2000, o governo federal implementou nas escolas o Programa de Informática de Educação Especial (PROINESP). Com isso, foram contempladas mais de duzentas escolas e capacitados mais de mil profissionais. Estes profissionais seriam os multiplicadores do processo. Contudo, considerando as dimensões continentais do Brasil, as metas desejadas não foram atingidas (Barwaldt, Santarosa \& Passerino, 2008).

Recentemente, o Projeto de Lei $n^{\circ} 3477 / 2020$ que dispunha sobre a garantia de acesso à internet, com fins educacionais, a alunos e a professores da educação básica pública não foi aprovado (Brasil, 2021b). Segundo o Censo Escolar do Instituto Nacional de Estudos e Pesquisas Educacionais Anísio Teixeira (Inep) as escolas municipais, mesmo em maior número no ensino fundamental, são as que menos dispõe de recursos tecnológicos para os alunos, como lousa digital (9,9\%), projetor multimídia $(54,4 \%)$, computador de mesa $(38,3 \%)$ ou portátil $(23,8 \%)$ e internet disponível para uso dos estudantes $(23,8 \%)$ (Brasil, 2021b).

Foi publicado o Decreto n 10.502/20 (Brasil, 2020), que buscava instituir a "Política Nacional de Educação Especial: Equitativa, Inclusiva e com Aprendizado ao Longo da Vida". A publicação do decreto reacendeu o debate sobre a educação especial e a educação inclusiva e, não obstante ter efeitos suspensos por meio de decisão judicial (Ação Direta de Inconstitucionalidade $\mathrm{n}^{\circ}$ 6590), promoveu o debate sobre o tipo de política educacional que se espera, no país, sob a ótica da inclusão (Brasil, 2021c).

Da decisão que suspendeu os efeitos do Decreto 10.502/20, vale destacar:

O paradigma da educação inclusiva é o resultado de um processo de conquistas sociais que afastaram a ideia de vivência segregada das pessoas com deficiência ou necessidades especiais para inseri-las no contexto da comunidade. Subverter esse paradigma significa, além de grave ofensa à Constituição de 1988, um retrocesso na proteção de direitos desses indivíduos. A Política Nacional de Educação Especial questionada contraria o paradigma da educação inclusiva, por claramente retirar a ênfase da matrícula no ensino regular, passando a apresentar esse último como mera alternativa dentro do sistema de educação especial. Desse modo, o Decreto $\mathrm{n}^{\circ} 10.502 / 2020$ pode vir a fundamentar políticas públicas que fragilizam o imperativo da inclusão de alunos com deficiência, transtornos globais do desenvolvimento e altas habilidades ou superdotação na rede regular de ensino (Brasil, 2021c, p. 31).

A densidade do tema demandou a realização de audiência pública com o fim de fomentar a participação social, possibilitando "reflexões qualificadas de pessoas e entidades que, embora fora da relação processual formal, trabalham ou atuam diretamente com o tema em discussão" (Brasil, 2021a, p. 6). Nesse cenário, a ideia de uma educação permanente no século XXI tem sido muito defendida e incentiva os trabalhos de natureza diversificadas, como o uso das metodologias ativas, 
possibilitando ao discente a construção de um pensamento crítico, para que possa avaliar as fontes digitais e o uso ético da informação, em prol de uma sociedade mais inclusiva (Fava, 2016). A inclusão escolar não pode ser vista como algo inconveniente, como algo registrado somente em documentos normativos, ela precisa de fato ser posta em ação nos ambientes escolares através da formação continuada para os professores. É necessário mudar os discursos de muitos profissionais da educação ao se referir à Educação Especial como algo existente somente na teoria, sem possibilidades práticas (Leite \& Braz, 2021).

O documento descritivo da BNCC (Brasil, 2018) determina as 10 competências gerais a serem desenvolvidas, a seguir na Figura 1:

Figura 1: Competências gerais da BNCC.

\section{COMPETENCIIAS GERAIS DA NOVA BNCC}

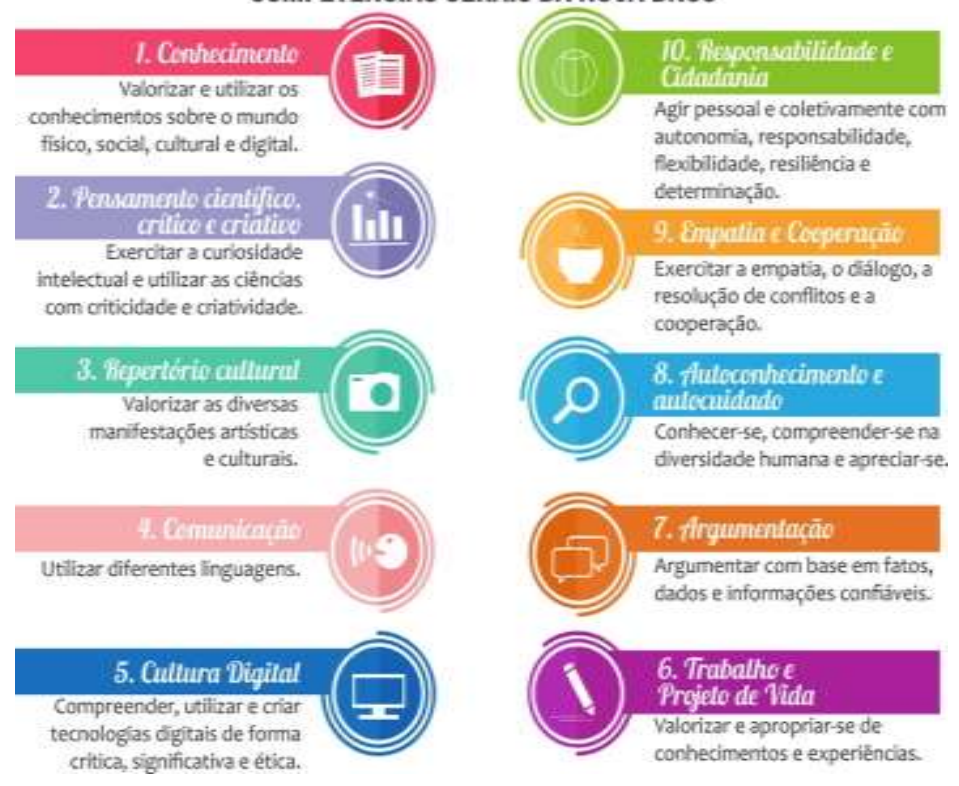

Início da descrição da imagem. Quadro com título: Competências Gerais da Nova BNCC. Em duas colunas, dispostas em numeração contínua, com acabamento colorido, são indicados: 1. Conhecimento. Valorizar e utilizar os conhecimentos sobre o mundo físico, social, cultural e digital. 2. Pensamento científico, crítico e criativo: exercitar a curiosidade intelectual e utilizar as ciências com criticidade e criatividade. 3. Repertório cultural. valorizar as diversas manifestações artísticas e culturais. 4. Comunicação: utilizar diferentes linguagens. 5. Cultura digital: compreender, utilizar e criar tecnologias digitais de forma crítica, significativa e ética. 6. Trabalho e projeto de vida: valorizar e apropriar-se de conhecimentos e experiências. 7. Argumentação: argumentar com base em fatos, dados e informações confiáveis. 8. Autoconhecimento e autocuidado: conhecer-se, compreender-se na diversidade humana e apreciar-se. 9. Empatia e cooperação: exercitar a empatia, o diálogo, a resolução de conflitos e a cooperação. 10. Responsabilidade e cidadania: agir pessoal e coletivamente com autonomia, responsabilidade, flexibilidade, resiliência e determinação. Fim da descrição. Fonte: https://bit.ly/31CrICO.

As competências apontadas pela BNCC sobre a maneira de lidar com a aprendizagem devem ser estimuladas, diante das dificuldades apresentadas em provas externas. Não se pode esperar que os adolescentes tenham interesse e curiosidade pela tecnologia somente quando chegarem nos cursos técnicos ou graduação, ou que passem a vida sendo apenas usuários passivos das diversas ferramentas tecnológicas presentes no nosso dia a dia. Quanto mais cedo as crianças tiverem contato com atividades que estimulem o pensamento lógico, as noções de abstração e algoritmo, entre outras, elas serão capazes de aplicar em contextos diversos (Pinto \& Nascimento, 2018). Sendo assim, nos tempos atuais, a ideia que permeia o uso da tecnologia é o Pensamento Computacional. Segundo Guarda e Crespo (2021, p.3) "neste contexto, podem ser destacados os aplicativos educacionais, como uma ferramenta para motivar os estudantes a buscar, pesquisar, gerar novos conhecimentos, trabalhar de forma cooperativa como uma estratégia para manter o educando na escola, não por obrigação, mas por motivação". 
Com a integração da cultura digital no currículo escolar, fomentada pela Base Nacional Comum Curricular (Brasil, 2018), propõe-se o uso da tecnologia e de recursos digitais para os processos educativos, de forma que os alunos se tornem protagonistas desse processo, e sejam estimulados para um maior engajamento e motivação no processo de ensino aprendizagem. Isso permitirá que o aluno seja inserido dentro da cultura digital e desta forma faça uso do pensamento computacional como possibilidade na resolução de problemas, partindo do concreto para o abstrato através das atividades propostas.

\section{Metodologia}

Como encaminhamento metodológico, emprega-se a pesquisa um levantamento bibliométrico realizado nas bases científicas do Google Acadêmico e da Web of Science, com as palavras-chaves: pensamento computacional, inclusão, tecnologia de informação e comunicação, pessoas com deficiências e altas habilidades. Estabelecemos como critério de busca a Lei de frequência de palavras de Zipf (Araújo, 2006), bem como os desdobramentos desta lei e suas aplicações nas principais áreas de conhecimento predominantes dos artigos. Posteriormente, com o intuito de selecionar e restringir os trabalhos publicados sobre a temática, delimitamos as buscas da produção acadêmica nas bases científicas do Google Acadêmico e da Web of Science, considerando os últimos cinco anos (período de 2017 a 2021) de publicações.

Será então apresentado um relato de experiência sobre um grupo de pesquisa que estuda o pensamento computacional e a inclusão das pessoas com deficiências e as altas habilidades em todas as modalidades de ensino. Este grupo surgiu a partir das reuniões que aconteceram remotamente no período da pandemia, tendo sido nomeado Tecnologias Computacionais no ensino e aprendizagem na ótica da Diversidade, Inclusão e Inovação (TeCEADI+), e cadastrado no Conselho Nacional de Desenvolvimento Científico e Tecnológico (CNPQ). Além disso, tal grupo foi instituído a partir da necessidade de discutir os pilares clássicos do pensamento computacional, sob a ótica da inclusão. Trata-se assim de um recorte dos debates ocorridos online, a partir das experiências identificadas como relevantes e aplicáveis, pelos alunos de mestrado e doutorado de diferentes áreas profissionais.

No Google Acadêmico foram pesquisadas as palavras em inglês, na data de 28/09/2021 e encontramos: computational thinking: 2.190 .000 artigos; inclusion: 5.230.000; information and communication technology: 5.660.000; people with disabilities: 2.720.000; e high abilities or giftedness: 93.400, sendo o somatório 15.893 .400 artigos. Quando realizada a mesma pesquisa em português, na mesma data, foram encontradas para o pensamento computacional: 79.300 artigos; inclusão: 2.220.000 artigos; tecnologia de informação e comunicação: 632.000; pessoas com deficiências: 677.000; e altas habilidades ou superdotação 22.000 artigos, sendo o somatório 3.630 .300 artigos.

Como novo resultado, para refinar a pesquisa buscou-se no Google Acadêmico, na mesma data, as seguintes palavraschave agrupadas em português: pensamento computacional e inclusão (encontram-se 36.200 artigos), pensamento computacional e tecnologia de informação (55.500 artigos), pensamento computacional e comunicação (60.200 artigos), pensamento computacional, pessoas com deficiências e altas habilidades (15.600 artigos), sendo o somatório 167.500 artigos. Em seguida, foi realizada a mesma pesquisa, em inglês alcançando os resultados para: computational thinking and inclusion (185.000), computational thinking and information technology (1.090.000), computational thinking and communication (783.000), computational thinking, people with disabilities and high skills (39.400), sendo o somatório 2.097 .400 artigos.

Os números de artigos encontrados indicam a farta produção de conteúdo relacionado às palavras-chave. Sendo assim, realizamos as strings com as palavras-chave: pensamento computacional, inclusão, tecnologia de informação e comunicação, encontrando como resultado 21.100 artigos em português; já em inglês foram encontrados 121.000 artigos. Foi acrescentada então mais uma palavra-chave: pensamento computacional, inclusão, tecnologia de informação e comunicação e pessoas com deficiências, totalizando em português 15.000 artigos, e em inglês 24.900 artigos. 
E dando prosseguimento à pesquisa foi realizada mais uma busca no Google acadêmico utilizando as palavras-chave: pensamento computacional, inclusão, tecnologia de informação e comunicação e pessoas com deficiências e altas habilidades ou superdotação. Em inglês encontramos 8.590 artigos, e em português 962 artigos.

Devido ao elevado número de artigos encontrados, e a fim de filtrar as informações e delimitar ainda mais a pesquisa, refizemos a busca no Google Acadêmico, na data de 29/09/2021, determinando um período de tempo dos últimos 05 anos (de 2017 a 2021) e encontramos os seguintes resultados com as palavras em Inglês: computational thinking, 108.000 inclusion 920.000, information and communication technology 1.330.000, people with disabilities 143.000 and high abilities giftedness 15.800, sendo o somatório 2.516 .800 artigos. Ao realizarmos a mesma pesquisa em português, na mesma data, foram encontradas para o pensamento computacional 15.900 artigos, inclusão 127.000 artigos, tecnologia de informação e comunicação 20.800; pessoas com deficiências 15.100; e altas habilidades ou superdotação 11.100 artigos, sendo o somatório 189.900 artigos.

Dando continuidade à pesquisa buscou-se no Google Acadêmico, na mesma data, as seguintes palavras-chaves agrupadas em português: pensamento computacional e inclusão (encontram-se 13.700 artigos), pensamento computacional e tecnologia de informação (16.000 artigos), pensamento computacional e comunicação (15.900 artigos), pensamento computacional, pessoas com deficiências e altas habilidades (5.810 artigos), sendo o somatório 51.410 artigos. Em seguida, foi realizada a mesma pesquisa, em inglês alcançando os resultados para: computational thinking and inclusion (19.700), computational thinking and information technology (32.500), computational thinking and communication (39.300), computational thinking, people with disabilities and high skills (17.200), sendo o somatório 108.700 artigos.

Mesmo delimitando a busca ao período de 2017 a 2021, encontramos um grande quantitativo de artigos, e assim, realizamos as strings com as palavras-chave: pensamento computacional, inclusão, tecnologia de informação e comunicação e como resultado obtivemos 12.700 artigos em português, e em inglês 17.200 artigos. Foi acrescentada então mais uma palavrachave: pensamento computacional, inclusão, tecnologia de informação e comunicação e pessoas com deficiências em português 5.840, e em inglês 17.100 artigos.

Então foi realizada, na mesma data, a última pesquisa no google acadêmico usando as strings, acrescentamos as palavras-chaves: pensamento computacional, inclusão, tecnologia de informação e comunicação e pessoas com deficiências e altas habilidades ou superdotação, em inglês encontramos 2.080, e em português 515 artigos. Sendo ainda um número alto de artigos a serem lidos, realizamos, então, a pesquisa considerando todos os campos na web of Science, no perído de 2017 a 2021, com as palavras-chave em inglês computational thinking and inclusion e encontramos 64 artigos.

Os artigos encontrados com as palavras-chaves citadas acima estão divididos nas seguintes temáticas: Education Educational Research- 24 artigos, Education Scientific Disciplines - 15 artigos, Computer Science Interdisciplinary Application - 8 artigos, Engineering Electrical Eletronic - 4 artigos, Engineering Multidisciplinary - 3 artigos; Computer Science Theory Methods - 5 artigos, Psychology Educational - 2 artigos, Computer Science Cyberretics - 1 artigo, Computer Science Information Systems - 1 artigo e Computer Science Artificial Inteligence - 1 artigo.

Optamos então por realizar a busca com Strings utilizando as seguintes palavras: computational thinking, inclusion and information and communication technology, e não encontramos nenhum artigo disponível, portanto, fizemos a busca com os Strings das palavras computational thinking and information and communication technology e encontramos 24 artigos.

Os artigos encontrados com as palavras-chaves citadas acima estão divididos nas seguintes temáticas: Telecommunications - 6 artigos, Engineering Electrical Eletronic - 5 artigos, Computer Science Information Systems - 4 artigos, Computer Science Interdisciplinary Applications - 2 artigos, Computer Science Theory Methods - 2 artigos, Chemistry multidiciplinary - 1 artigo, Computer Science artificial Intelligence - 1 artigo, Computer Science Software Enginnering - 1 artigo, Communication - 1 artigo e Computer Science Hardware Architecture - 1 artigo. 
Posteriormente, seguem-se as consultas com os strings das seguintes palavras: computational thinking and people with disabilities. Nós não encontramos nenhum artigo sobre o assunto. E em seguida, com os Springs: computational thinking and high abilities giftedness. Também não foi encontrado nenhum artigo demonstrando a necessidade de pesquisas sobre a temática.

O Quadro 1 resume as buscas realizadas na base de dados científica da Web of Science, tendo sido utilizadas as iniciais das palavras para confeccioná-lo: Computational Thinking (CT), Inclusion (IN), Information and Communication Technology (ICT), People with disabilities (PD), High Abilities Giftedness (HAG).

Quadro 1: Base de dados pesquisados na Web of Science.

\begin{tabular}{|c|c|c|c|c|}
\hline $\mathrm{CT}+\mathrm{IN}$ & $\mathrm{CT}+\mathrm{IN}+\mathrm{ICT}$ & $\mathrm{CT}+\mathrm{ICT}$ & $\mathrm{CT}+\mathrm{PD}$ & $\mathrm{CT}+\mathrm{HAG}$ \\
\hline 64 artigos & 0 & 24 & 0 & 0 \\
\hline
\end{tabular}

Fonte: Arquivo do Grupo de Pesquisa.

Após a busca na base de dados, Web of Science, foram localizados 88 artigos, um número satisfatório de trabalhos para realizar um estudo exploratório dos resumos, a partir de uma narrativa qualitativa. Nesse sentido, é válido destacar que a temática do trabalho é inovadora, já que, quando realizamos as strings com as palavras-chave não conseguimos encontrar nenhum trabalho publicado.

\section{Resultados e Discussão}

No contexto da educação, a utilização do pensamento computacional (PC), pode ajudar crianças e jovens a aprender técnicas de resolução de problemas que podem ser úteis não apenas no escopo da informática, mas para os vários desafios que vão enfrentar ao longo da vida. Esta visão defende que a cultura do computador ajuda a sociedade não somente a aprender, mas especialmente oferece uma nova maneira de aprender a aprender (Lu \& Fletcher, 2009; Papert, 1980).

Considerando os avanços tecnológicos atuais, que revolucionaram as mídias de comunicação e educação, é possível afirmar que inúmeras são as possibilidades de criação de tecnologias educacionais, capazes de correlacionar interdisciplinarmente milhares de informações em tempo real. Com isso, os ambientes/plataformas/aplicativos educacionais são representativos do poder da tecnologia para dar apoio às práticas pedagógicas inclusivas.

Conforme Almeida e Prado (1999, p.1, como citado em Barbosa \& China, 2017, p.10):

Hoje é consenso que as novas tecnologias de informação e comunicação podem potencializar a mudança do processo de ensino e de aprendizagem e que, os resultados promissores em termos de avanços educacionais relacionam-se diretamente com a ideia do uso da tecnologia a serviço da emancipação humana, do desenvolvimento da criatividade, da autocrítica, da autonomia e da liberdade responsável (Almeida \& Prado, 1999, p.1, como citado em Barbosa \& China, 2017, p.10).

De acordo com estudos realizados por Barcelos e Silveira (2012), uma estratégia para inserção do Pensamento Computacional na Educação Básica deve ocorrer através de disciplinas pré-existentes no atual currículo, como por exemplo a Matemática. A Sociedade Internacional de Tecnologia em Educação (ISTE) também realizou um levantamento com diversas propostas de adoção de forma Pluri, Multi e Transdisciplinar (Brackmann, 2017), assim como outras propostas similares são relatadas por Barr e Stephenson (2011).

Nas reuniões que ocorreram durante a pandemia, o Grupo de pesquisa TeCEADI+ estabeleceu a figura 3 como a logomarca que o representa retratando a diversidade, os quatro pilares do pensamento computacional e a inclusão de todos. Segundo Guarda e Crespo (2020): 
O Pensamento Computacional pode ser compreendido como um approach voltado para a resolução de problemas explorando processos cognitivos, pois discutem a capacidade de compreender as situações propostas e criar soluções através de modelos matemáticos, científicos ou sociais para aumentar nossa produtividade, inventividade e criatividade (Guarda \& Crespo, 2020, p.1463).

Tanto as atividades desplugadas quanto plugadas podem ser utilizadas para desenvolver os quatro pilares que fundamentam o pensamento computacional, que são divididas segundo Ramos (2014) em: decomposição, que é a capacidade de dividir um problema em partes menores facilitando a resolução de um problema complexo; reconhecimento de padrão, que é a habilidade de reconhecer padrões separando em grupos de similaridades para identificar comportamentos específicos; abstração que é focar no que realmente importa deixando de lado os detalhes para encontrar soluções mais específicas para o problema; e pensamento algoritmo que é a capacidade de desenvolver e seguir uma série de passos ordenados com o objetivo de chegar a uma solução para um problema.

O pensamento computacional é uma habilidade presente em processos como a leitura, escrita e matemática, é uma habilidade analítica que vai sendo construída durante o desenvolvimento cognitivo das crianças para encontrar os passos necessários para resolver os problemas (algoritmos). Porém, como outras habilidades, se o seu desenvolvimento não for incentivado, pode ser perdido ao longo da vida (Ramos, 2014, p. 34).

As discussões provenientes desses pilares e habilidades provocaram uma reformulação da prática educacional a partir das teorias que envolvem o Pensamento Computacional, resultando em atividades diversificadas nos diferentes níveis da educação básica e do ensino superior. A aplicabilidade desse conhecimento incide diretamente no desenvolvimento e qualidade de vida desses sujeitos, pessoas que em algum momento passam por dificuldades acentuadas na aprendizagem e precisam de estratégias inclusivas adequadas às suas necessidades educacionais. Algumas dessas atividades foram desenvolvidas no grupo de pesquisa e apresentadas no (Senid) Seminário Internacional de Inclusão Digital (2021), publicadas em artigos, repositórios, congressos e minicursos.

Figura 2: Logo que representa o grupo de pesquisa TeCEADI+.

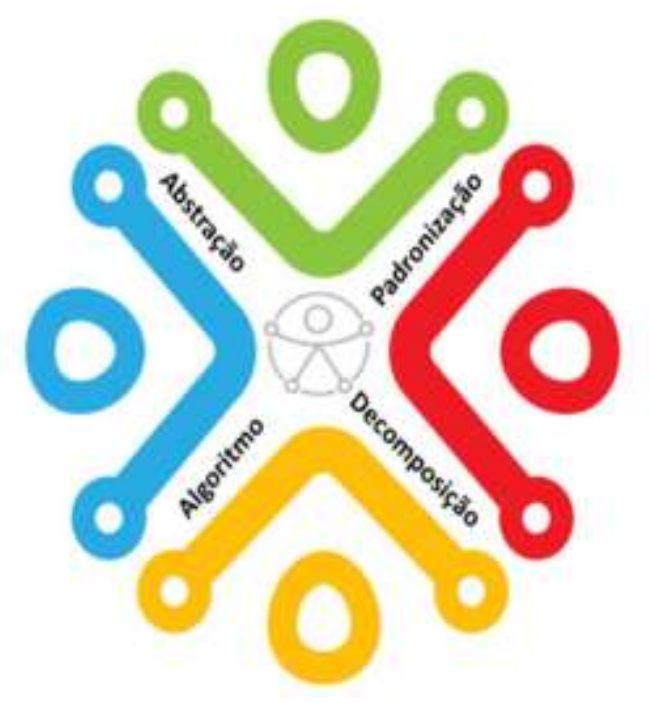

Descrição: A imagem é um desenho gráfico, semelhante a representação de uma pessoa com os braços abertos. Existem quatro figuras com esse mesmo desenho, cada uma de uma cor (azul, verde, vermelho e amarelo), que estão organizadas em formato circular, como se essas pessoas estivessem de frente umas para as outras. Na área em que os braços se aproximam, é possível visualizar uma passagem onde se lê: abstração, padronização, algoritmo e decomposição. Ao centro da figura, temos o símbolo internacional de acessibilidade. Fonte: Arquivo do Grupo de Pesquisa. 
As instituições de ensino (tanto do nível básico, quanto do superior) precisam considerar as tecnologias digitais e os reflexos destas nos processos de ensino e de aprendizagem. Isso porque, assim como o aluno já não é mais o mesmo, a sociedade constrói teias cada vez mais complexas de relações - ao exigir, corretamente, um novo tipo de ensino.

Com isso, é preciso rever o papel da instituição escolar e de sua política institucional. Também é preciso conhecer o aluno, como um todo (em potenciais, interesses e vulnerabilidades). A partir disso torna-se indispensável a análise de ferramentas que aproximem a sala de aula tradicional dos ambientes e das tecnologias digitais.

Assim, surgiram as primeiras perguntas quanto às habilidades necessárias nesse novo contexto de ensino que abrangem: a) a existência de ferramentas tecnológicas disponíveis em salas de aula; b) o preparo de professores para as novas tecnologias; c) a existência de um projeto pedagógico integrador e claro o suficiente para nortear o futuro das disciplinas e, ainda, d) a dinâmica da organização do tempo para alunos e professores.

\subsection{O Pensamento Computacional como ferramenta de auxílio a aprendizagem do discente com impedimento auditivo}

De início, é importante considerar que o impedimento auditivo é plural e pode decorrer de uma multiplicidade de fatores, dentre os quais destacam-se: genética, comorbidades perinatais, traumas, medicação ototóxica, infecções, deficiências nutricionais e exposição a ruídos. Desse modo, as abordagens de acompanhamento multidisciplinar podem abranger: tecnologias auditivas (na forma de aparelhos auxiliares, implantes cocleares e aparelhos auditivos), língua de sinais, substituições sensoriais (como leitura labial) e, ainda, terapia de reabilitação, que pode ser auditiva e de fala (WHO, 2021).

Com isso, é comum que o professor se depare com variações de necessidades do discente com impedimento auditivo que dependerão do momento em que ocorreu a sua perda, o tipo de instrumentalização tecnológica recebida pelo aluno, bem como a fluência ou não em língua de sinais. Por isso é importante frisar o acompanhamento interdisciplinar do aluno, já que nem toda demanda do discente será suprida pelo professor. Independente destas variáveis, é reconhecido que:

A perda auditiva pode ter um impacto duradouro sobre os resultados acadêmicos de um indivíduo. Caso não sejam tratados em tempo hábil, aqueles com perda auditiva têm sua performance escolar reduzida, progressão mais lenta em sua vida acadêmica, um maior risco de evasão escolar e menor probabilidade de chegar ao Ensino Superior quando comparados com seus pares ouvintes (WHO, 2021, p. 46, tradução nossa). ${ }^{1}$

Um primeiro passo possível é incrementar os recursos visuais para o trabalho dos alunos surdos quanto às habilidades necessárias ao desenvolvimento dos pilares relacionados ao PC. Com isso, poderíamos ter uma maior concentração visual como recurso para auxiliar na abstração. Assim, é possível introduzir conceitos da ciência da computação com as chamadas atividades desplugadas (Bell, Witten, Fellows, Adams \& McKenzie, 2011), que consistem em vivenciar os conceitos a partir de situações e atividades concretas. A utilização dessa estratégia beneficia diretamente os discentes surdos que nas séries iniciais precisam dessas situações concretas para se apropriar de conceitos e conteúdo.

Todas essas habilidades poderão ser desenvolvidas e estimuladas de forma lúdica, com atividades desplugadas e com o uso de smartphones, tablets, notebooks e computadores através do uso de programas voltados para o ensino de programação como o Turtle Blocks ${ }^{2}$, Scratch ${ }^{3}$ e o Scratch $\mathrm{Jr}^{4}$. Essas opções exploram as cores, formas e movimento, ultrapassando a mera utilização de equipamentos e contemplando a forma como se faz uso desses para o crescimento humano, pois auxiliam no letramento visual.

\footnotetext{
${ }^{1}$ Hearing loss can have a long-lasting impact on the academic outcomes of an individual. Unless addressed in a timely manner, those with hearing loss have reduced school performance, slower progression through the academic system, a greater risk of dropping out of school, and lower likelihood of applying for higher education, compared with their hearing peers.

${ }^{2}$ https://turtle.sugarlabs.org/

${ }^{3}$ https://scratch.mit.edu/

${ }^{4}$ https://www.scratchjr.org/
} 
Uma sugestão de jogo pensado pelo grupo, inspirado no trabalho de Andrade et al. (2013) é a adaptação do jogo "Cara a Cara" para um grupo de alunos surdos, sinalizantes, que poderiam identificar seus colegas de classe a partir de suas características físicas e sinais (em Libras). Os alunos poderiam ser orientados a padronizar a identificação dos sinais dos colegas (se são feitos, por exemplo, com uma única mão ou com as duas), associar características físicas ao sinal correspondente em Libras, criando padrões de resposta que permitam a conclusão do jogo, com a identificação do aluno selecionado para identificação. O jogo permite, ainda, que os alunos verifiquem quais características físicas mais se repetem, quais padrões produzem melhores resultados na dinâmica da disputa e, assim, estariam trabalhando, de forma lúdica, conceitos interdisciplinares do PC.

\subsection{O Pensamento Computacional como ferramenta de auxílio a aprendizagem do discente com Deficiência Intelectual} (DI)

Um estudo mais aprofundado das pedagogias críticas pode trazer à tona os movimentos que ressignificam as formas de se aprender e ensinar na escola, respeitando-se a diversidade, trabalhando seu currículo, adaptando-o quando necessário. O que se ensina deveria estar totalmente atrelado às necessidades de quem aprende, assim como deve-se respeitar as limitações do campo cognitivo e social dos estudantes (Stainback \& Stainback, 1999).

Teorias pós-modernas que amparam o paradigma inclusivo leva-nos a pensar coletivamente, viver em comunidade respeitando as diferenças entre seus pares, e a partir de então desenvolver um ambiente propício à aprendizagem real e inclusiva, principalmente quando se trata de pessoas com DI (Stainback \& Stainback, 1999). Portanto, discutir, falar uns aos outros sobre as peculiaridades de seus alunos é de suma importância para o desenvolvimento de estratégias tecnológicas para atender também a este público.

As escolas demonstram dificuldades em atender alunos com especificidades educacionais, principalmente cognitiva, tendo um enfoque na valorização do saber. Dessa forma, um trabalho de colaboração entre os profissionais que atuam neste âmbito é fundamental para que se desmistifique a forma de se atender a demanda do ensino inclusivo (Barab \& Roth, 2006).

O ambiente em termos de redes de recursos que se estendem no tempo e no espaço e podem incluir como possibilidades perceptuais e cognitivas que coletivamente formam a rede para determinados conjuntos de metas. Uma perspectiva ecológica sobre o conhecimento argumenta que saber é o processo de envolver com sucesso um sistema intencionalmente limitado de tal forma que objetivos específicos são alcançados (Barab e Roth, 2006 como citado em Jackson, 2013, p. 4)

Nessa perspectiva ecológica do ensino, deve-se dar um trabalho colaborativo entre os docentes potencializando-o, assim como intervir conscientemente no processo educacional, descrevendo metas claras que deverão culminar em resultados possíveis. Neste ínterim, as discussões sobre as características de sujeitos comprometidos cognitivamente, as diferentes formas de aprender podem indicar possibilidades, estratégias que sejam funcionais. Currículos que atendam ao aluno, amparados por estratégias que o leve a pensar durante a execução das atividades. O uso do PC nesse sentido pode contribuir significativamente para a evolução cognitiva dos sujeitos, assim como acontece com pessoas neuro típicas.

Alunos com DI precisam de metodologias diferenciadas, e ficou evidente em função da pandemia, a enorme dificuldade dos agentes da educação encontrarem caminhos que facilitem a aprendizagem desses sujeitos. As limitações que possuem passam principalmente pela cognição, quando não associadas a outras comorbidades, desta forma é preciso inserir muitos e variados estímulos (Gomes, 2021). As características da DI, ou mesmo de pessoas que apresentam comportamento autístico, precisam de atividades que facilitem suas conexões cerebrais para criar novas rotas de aprendizagem de acordo com a neurociência. 
O PC inicialmente pode ser trabalhado com esses sujeitos usando os quatro pilares de forma desplugada principalmente (Bell et al., 2011). Ao observar o sujeito, suas características, rotas de aprendizagem mais alinhadas com o seu estilo de aprendizagem. Então inserir atividades cognitivas reforçadas pela repetição, até que apreenda uma das habilidades do PC, coloque em prática em atividades cotidianas é apenas uma das possibilidades de trabalho. Essas atividades devem ser aplicadas de forma lúdica, com jogos variados (Brackmann, 2017). Exemplificando, como um simples amarrar cadarços, um passo a passo desse procedimento, por vezes muito complexo para eles, auxilia na consolidação e aquisição desses processamentos internos e cognitivos com a aplicabilidade da decomposição.

Contudo, o maior entrave na educação de alunos com deficiência intelectual e neuro atípicos se dá no nível da abstração. Estudos realizados por Katz e Rubin (2000), precursores da "neuróbica", demonstraram que exercícios cerebrais explorando o sistema sensorial, estimulando padrões de atividades neurais potencializam a formação de conexões e engramas cerebrais em diferentes áreas do cérebro, maximizando o desenvolvimento nas atividades escolares e da vida diária (Comin \& Silva, 2020).

As pessoas com DI precisam ser atendidas de acordo com as habilidades que menos estiverem desenvolvidas. As intervenções pedagógicas devem proporcionar o apoio intermitente, limitado, extensivo ou generalizado, necessário para o desenvolvimento desses sujeitos (Glat, 2007). Também é relevante observar que a pessoa com DI pode apresentar alguma dificuldade na aprendizagem em níveis de comprometimento diferenciados, dificuldade em compreender conceitos abstratos, considerável dificuldade na generalização e aplicação do aprendizado, no funcionamento deficitário das funções de memória, na concentração, na atenção, na resolução de problemas e outros (Monteiro, 2017).

De modo que se considerarmos apenas esses dados, será comum presenciarmos situações em que as pessoas com DI serão privadas de atividades que envolvam o exercício das funções psíquicas de ordem superior (isto é, aquelas que se relacionam com os simbolismos de segunda ordem, como: o raciocínio, o planejamento, a memória e a linguagem oral ou escrita), devido à falsa crença de que "elas não aprendem". Incorrendo nesse erro da privação, condicionamos a pessoa com DI a não avançar em níveis de desenvolvimento cognitivo (Glat, 2007).

Logo a proposta do uso do Pensamento Computacional nesse sentido deve usar primeiramente o conhecimento sobre a pessoa com deficiência, a fim de perceber a intensidade e proporcionalidade em que se poderá aplicar algum pilar do Pensamento Computacional. As atividades deverão ser adaptadas de acordo com o sujeito, ampliando-se as possibilidades e graus de dificuldades, ao passo em que se percebe a evolução dentro de uma questão trabalhada. As pessoas com uma desvantagem intelectual, por exemplo, têm dificuldades especiais nas áreas de organização e categorização de experiências, tais como: tempo, espaço, qualidade, quantidade e causa; uso de informações estruturadas para realizar "operações de pensamento" uma habilidade em representar a realidade por meio de símbolos pode ajudar, assim como o exemplo de atividade prática funcional descrita no trabalho.

Para alguém ser capaz de ir ao cinema de modo independente, é necessário considerar o horário da sessão e quanto tempo levará para chegar até lá (conceito de tempo), onde este está localizado (conceito de espaço), quanto custa a entrada (conceito de quantidade) e se o filme é bom (conceito de qualidade). Esta pessoa precisa considerar essas informações em seu pensamento (operações de pensamento) e decidir se pode atendê-las ou escolher as alternativas existentes. É preciso ainda que essa pessoa use sua habilidade para interpretar símbolos para encontrar informações referentes ao assunto nos jornais ou internet, por exemplo, (habilidade de representar a realidade por meio de símbolos).

Nosso intelecto está de tal modo implicado que não paramos para pensar nas mais simples decisões do cotidiano. No entanto, para a pessoa com DI, uma simples ação, como as descritas acima, torna-se algo complexo de ser alcançado. Nesse sentido, reafirmamos que a prática pedagógica que não envolva a resolução de problemas cotidianos, dificilmente promoverá o alcance de níveis elevados de desenvolvimento pela pessoa com deficiência intelectual. 
É preciso que o educador, dentro dessa conjuntura, ressignifique a sua forma de conceber o processo de ensinoaprendizagem desses alunos, usando o Pensamento Computacional para potencializar o ensino. O educando pode não aprender num dado momento ou com aquela estratégia, mas isso não quer dizer que ele não vá aprender no seu ritmo e em consonância com o nível de desenvolvimento em questão.

\section{3 O Pensamento Computacional como ferramenta de auxílio a aprendizagem do discente com impedimento visual}

A visão é uma importante ferramenta de ligação dos indivíduos com o mundo objetivo, pelo fato de proporcionar informações constantes de modo imediato, permitindo ainda que os elementos sejam apreendidos de maneira integrada, não fragmentada (Santin \& Simmons, 1996). Dessa forma, a presença da visão tem grande influência na percepção da realidade e na organização interna que a pessoa constrói do mundo. Por outro lado, por mais que sua ausência possa limitar a interação com o ambiente, o organismo dispõe de outros caminhos que podem suprir ou complementar informações de nature za visual.

Em virtude da capacidade do cérebro de mudar sua organização molecular e funcional como resposta, entre outros, à privação ou à lesão (neura plasticidade cerebral), os neurônios são capazes de criar novas conexões e novos caminhos com diferentes funções no córtex para suprir a falta de visão, promovendo importantes mudanças anatômicas e funcionais que resultam no desenvolvimento de habilidades compensatórias, como aprimoramento do tato, audição e do olfato (Mašić, Šečić, Bobić \& Femec, 2020). Além disso, funções cognitivas relacionadas à memória e à linguagem também são aprimoradas. Em estudo de Bauer et al., (2017), são descritas diversas mudanças anatômicas e funcionais no cérebro de pessoas nascidas com cegueira que não são observadas em pessoas videntes.

As existências de referidas modificações estruturais têm implicações na forma como pessoas cegas aprendem, desenvolvem conceitos e dão sentido aos conteúdos dos diferentes componentes curriculares, na medida em que seus principais meios de obtenção das informações passam a ser a audição e o tato. Assim, embora uma deficiência visual não implique, por si só, alterações que impeçam a aprendizagem, é importante perceber que, por terem um equipamento sensorial diferente, estudantes com deficiência visual organizam suas percepções de mundo e desenvolvem suas aprendizagens de maneira particular, diferente da dos videntes.

Desse modo, o despreparo de professores pode resultar na adoção de procedimentos educacionais tendo como parâmetro as formas de aprender do vidente, o que poderia significar uma recusa à deficiência e à percepção das possibilidades e limitações do cego, podendo causar restrições na aprendizagem desses alunos (Nunes \& Lomônaco, 2008). Assim, é importante que os professores compreendam as particularidades do modo de aprender desses estudantes, planejando o currículo e as situações de aprendizagem levando em conta tais especificidades. O desenvolvimento das habilidades do pensamento computacional junto a estes estudantes se insere nesse contexto, na medida em que é importante que professores conheçam os recursos disponíveis para tal, bem como as especificidades desse tipo de prática pedagógica.

Morrison et al. (2020) colocam que por mais que venham sendo criadas políticas em todo o mundo para promover o engajamento de crianças na programação de computadores e no desenvolvimento do pensamento computacional, atualmente não existem ferramentas para ensinar conceitos de programação e do PC para crianças do Ensino Fundamental com deficiência visual que sejam adequadas à idade. Já Riazy, Weller e Simbeck (2020) analisaram dezessete ambientes introdutórios de programação, bem como sua usabilidade para pessoas com deficiência visual, concluindo que nenhum deles era adequado para pessoas com esse tipo de impedimento, na medida em que utilizavam estruturas baseadas em arrastar e soltar blocos, não apresentavam texto alternativo ou demandam a utilização do mouse.

Por outro lado, é possível identificar esforços no sentido de construir novas linguagens e ambientes de programação, ou realizar adaptações nos já existentes, com o objetivo de fornecer acessibilidade para pessoas com deficiência visual. Hadwen-Bennett, Sentance e Morrison (2018), por exemplo, destacaram o fato de que muitos ambientes de programação 
modernos são inacessíveis para alunos com deficiência visual, sendo difícil ou impossível usá-los com um leitor de tela. No entanto, os autores identificaram estratégias que vêm sendo empregados para tornar a aprendizagem de programação acessível a estudantes com deficiência visual, como o uso de artefatos físicos (programação física), o uso de feedback auditivo e tátil, e os esforços para criação de linguagens de programação acessíveis baseadas em texto ou em blocos.

Os esforços mencionados no sentido de tornar o ato de programar acessível a estudantes com deficiência visual indicam algumas das possibilidades para o trabalho das habilidades do pensamento computacional com esses alunos. Através do uso de diferentes recursos e tecnologias, é possível desenvolver situações de aprendizagem nas quais os estudantes possam ser estimulados a solucionarem problemas utilizando aplicativos, softwares ou outros recursos computacionais. Rezende e Pinto (2021) identificaram, por exemplo, uma série de tecnologias digitais empregadas em objetos de aprendizagem da matemática voltados para estudantes com deficiência visual. Para além da programação de computadores, recursos desta natureza representam importantes ferramentas pedagógicas para o desenvolvimento de conteúdos escolares e, ainda, das habilidades do pensamento computacional.

Além disso, considerando que o PC é o processo de pensamento envolvido na formulação de um problema e na expressão de sua solução de modo que um computador possa efetivamente executar (Wing, 2010), a criação de atividades que objetivem estimular o desenvolvimento de tal processo de pensamento não envolve necessariamente o uso do computador, mas sim a possibilidade de resolução através de um recurso computacional. Nesse sentido, são relevantes os processos cognitivos relacionados à abstração e decomposição de modo que se permita a resolução de problemas utilizando estratégias algorítmicas, dentre outras habilidades (Barcelos, Muñoz, Acevedo, \& Silveira, 2015). Assim, a criação de atividades desplugadas acessíveis que explorem os demais sentidos também é viável para o desenvolvimento das habilidades do PC junto a estudantes com deficiência visual.

Por fim, a partir da realização de adaptações como uso de material tátil ou recursos sonoros, o processo de resolução de problemas pode ser explorado tanto em uma abordagem desplugada inicialmente, possibilitando o desenvolvimento de habilidades relacionadas à abstração, decomposição, reconhecimento de padrões e algoritmos, quanto plugada, possibilitando o uso de softwares e o trabalho de elementos de modelagem no processo de resolução.

\subsection{O Pensamento Computacional como ferramenta de auxílio a aprendizagem do discente com transtorno do Espectro} autismo

Ampliando o olhar para o discente com Transtorno do Espectro Autista (TEA), que particularmente pode ter ou não deficiência intelectual (American Psychiatric Association, 2013), há de se pensar na possibilidade de favorecer suas habilidades, visto que sujeitos que fazem parte deste grupo, apresentam, na maioria das vezes. Discentes com TEA são diferentes uns dos outros, apresentam demandas e necessidades específicas e singulares. Por este motivo, é importante pensar e incluir tarefas de cunho baseadas em necessidades específicas como: hiperfoco - que é o interesse demasiado em determinado assunto; responsividade sensorial - que está diretamente relacionada à forma como esse indivíduo recebe informações do ambiente e as acomoda; habilidades - assuntos em que apresentam conhecimento acima da média e atrasos - que estão relacionados às suas dificuldades específicas e vão de aprendizado a questões voltadas à interação social (Joia \& Braz, 2021).

Considerar uma pessoa com TEA é considerar uma variabilidade de necessidades particulares advindas da singularidade de cada sujeito, as habilidades, nível de severidade e o tipo de apoio que cada um necessitará em determinadas vivências sociais, cas, trabalho, social e escolar (Beytien, 2011; Leon \& Fonseca, 2013) Neste caso, antes de promover uma proposta pedagógica direcionada para este público, há de se compreender cada um a quem é dirigido e avaliar suas habilidades e suas necessidades de aprendizagem. Sujeitos com TEA apresentam necessidades pedagógicas e limites específicos, contudo 
uma demanda de aprendizado em determinadas áreas que muitas vezes ultrapassa o conteúdo e aprendizado assimilado em sala de aula.

Considerando o pensamento computacional como uma perspectiva interdisciplinar, trabalhado junto às disciplinas já existentes no currículo, a promoção de aprendizagem será aliada à construção de conhecimentos de diversos conteúdos, abrindo a possibilidade de aprendizagem mais dirigida à necessidade de cada sujeito aqui sinalizado (França \& Tedesco, 2015). É verdade também que alunos com TEA também são aprendizes autônomos em diversas situações como: computacional, línguas e temas mais específicos voltados à área da saúde, natureza e física.

Assim, quando falamos em crianças autistas, estamos pensando na sua forma natural de aprendizagem, isto é, ela desconhece as regras sociais e gramaticais, mas se torna capaz de aprender se forem incentivadas para tal e quanto mais cedo esse processo acontecer maiores serão as chances de desenvolvimento dessas crianças.

Entende-se então, que o pensamento computacional não atinge somente o aprendizado de recursos digitais, mas do uso de ferramentas computacionais assim como o aprendizado dos fundamentos da computação, com isso, há uma característica específica de atingir o aluno com TEA uma vez que é de seu interesse o tema e a diversidade de suportes pedagógicos que os quais vai oferecer. Alunos com TEA apresentam dificuldades específicas em abstrair informações de textos e falas, dificuldade atrelada à Teoria da Mente (Lima, 2012) que no Pensamento Computacional irá ter auxílio dante da competência Decomposição, onde um problema complexo pode ser dividido em partes menores para facilitar sua solução e com isso minimizar a ansiedade frente à desafios e levá-lo e uma resposta mais concreta e autônoma.

Um exemplo disto são o uso dos aplicativos e tablets para assimilação menos contextual e mais concreta do conteúdo e assimilação através de interesses restritos e de melhor resolução pelo aluno. Também com o uso de ambientes visuais de programação, assim como promover determinadas habilidades pela lógica da programação. Estas atividades irão estimular o pensamento computacional, motivar o aluno, ampliar suas trocas com os demais e despertar seu interesse por assuntos que antes poderiam ser excluídos pelo próprio aluno. Assim, a aprendizagem se torna mais significativa pois os interesses dos estudantes são levados em conta (França \& Tedesco, 2015).

\subsection{O Pensamento Computacional como ferramenta de auxílio a aprendizagem do discente com Altas Habilidades ou Superdotação (AH ou SD)}

Os alunos com $\mathrm{AH}$ ou SD demonstram potencial elevado em qualquer uma das seguintes áreas, isoladas ou combinadas: intelectual, acadêmica, liderança, psicomotricidade e artes, além de apresentar grande criatividade, envolvimento na aprendizagem e realização de tarefas em áreas de seu interesse (Brasil, 2008, p. 9). E em consonância com a Lei 13.234 de 2015, municípios, estados devem proceder à identificação, cadastramento e atendimento a esse público. Assim como catalogar esses estudantes (Brasil, 2015).

As Pessoas com AH ou SD apresentam características que lhe são muito próprias e dentre as quais podemos destacar, por exemplo: leitura precoce e de um número muito grande de livros; senso de humor, de ética e de justiça muito desenvolvidos, associação a pessoas muito mais velhas ou muito mais novas do que elas; tendência ao perfeccionismo e nível de autoexigência muito elevado; interesses diferentes aos dos seus pares; independência, autonomia, preferência por trabalharem/estudarem sozinhas. Comportamentos observáveis que por vezes podem levar os profissionais da educação ao erro, ao não perceberem a importância de potencializarem o ensino com estratégias enriquecedoras que direcionam o aprendizado desses sujeitos (Renzulli, 1986).

Dessa forma, o estigma que envolve a identificação e trabalho com esse alunado é significativamente prejudicial ao desenvolvimento holístico desses sujeitos, já que o pensamento errôneo de que não precisam de ajuda pedagógica para desenvolverem e potencializarem seus saberes é uma constante no meio escolar. O PC tem muito a contribuir para o 
desenvolvimento do aprendizado desses sujeitos. Uma organização de procedimentos que visam auxiliar o ensino e a aprendizagem quando bem geridos e motivados por seus interesses de estudo.

A resolução de situações problemas de acordo com o interesse do aluno contribui significativamente para o desenvolvimento de habilidades de reconhecimento de padrões, para o desenvolvimento do raciocínio por meio dos quatro pilares, que são: decomposição, reconhecimento de padrões, abstração de um problema e algoritmos; com atividades práticas na resolução de problemas, muito comum em atividades "makers", na resolução de problemas cotidianos, de forma prática. Uma sistematização do conhecimento que sigam os seguintes passos:

Figura 3 - A abordagem do Pensamento Computacional na Superdotação.

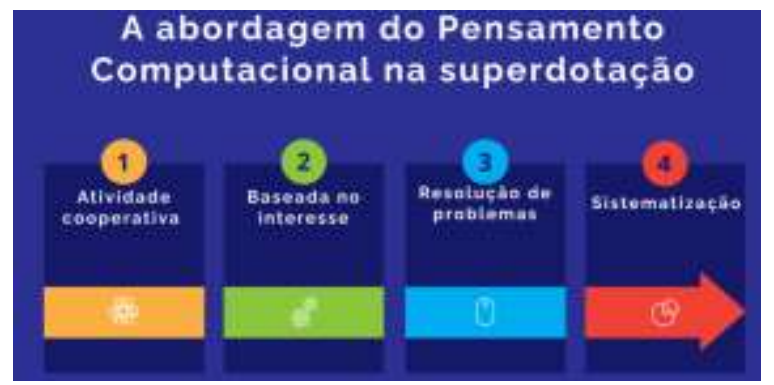

Descrição: Slide com o fundo na cor azul. Ao centro e com letras na cor branca, o título "A Abordagem do Pensamento Computacional na Supedotação". Abaixo, quatro retângulos com destaques dos números inseridos em círculos coloridos. O número 1, na cor laranja, corresponde ao subtítulo "Atividade cooperativa". O número 2, na cor verde, com o subtítulo "Baseada no interesse". O número 3, em um círculo azul, com o subtítulo "Resolução de problemas". O número 4, dentro do círculo vermelho, o subtítulo "Sistematização". Na base da figura, há uma seta apontando para a direita, que transpassa todos os retângulos, transmitindo a ideia de continuidade das fases citadas. Fim da descrição. Fonte: Arquivo do Grupo de Pesquisa TeCEADI+.

O uso do PC com esses alunos deve proporcionar a organização de um tempo para projetos de aprendizagem que contemplem o trabalho cooperativo, a resolução de problemas reais e a constante mediação dos processos cognitivos. Possivelmente um currículo engessado não favorece um trabalho diferenciado com esse público (Dantas, 2019). Contudo é preciso oferecer momentos em que a abordagem do PC possa ser vivenciada em grupos de estudo por interesses para resolverem situações problemas, e consequentemente sistematizar suas aprendizagens de forma a replicar esse saber para outras situações.

\section{Conclusão}

A educação inclusiva deve proporcionar o desenvolvimento de competências nas mais diferenciadas áreas, dessa forma o Pensamento Computacional tem muito a contribuir com a educação de pessoas com deficiência e altas habilidades ou superdotação para enriquecer e potencializar as estratégias pedagógicas.

Com efeito, não existem receitas prontas para se trabalhar com a diversidade encontrada nas escolas. O estudo trouxe algumas possibilidades de intervenção usando o Pensamento Computacional numa abordagem inclusiva, com o objetivo de trazer à tona as experiências de especialistas na área de ensino e diversidade, com o enfoque na tecnologia e no uso do Pensamento Computacional como estratégia de enriquecimento e complementação das atividades cognoscentes, baseadas em experiências e pesquisas bibliométricas.

Com isso, a contribuição para o campo de pesquisa e para a reflexão da prática pedagógica junto a esses sujeitos tende a ampliar as discussões no âmbito educacional, seja este formal ou informal. Incluindo-se centros de atendimento educacional 
especializado, laboratórios de enriquecimento curricular e a sala de aula regular, atendendo a BNCC quanto ao uso do Pensamento Computacional nas escolas para todos os alunos.

$\mathrm{O}$ artigo procurou evidenciar as diversas peculiaridades que existem e necessitam ser discutidas, estudadas, adaptadas para o uso do Pensamento Computacional sob a ótica da inclusão. No trabalho vários pontos foram elencados e para cada ponto torna-se necessário perceber o outro, ter empatia, para saber como, quando e de que forma os quatro pilares do Pensamento Computacional podem ser trabalhados nestes grupos. Resignificar o conceito bem como a sua prática sob a ótica inclusiva é necessário para termos uma amplitude na forma e conteúdo do como aplicar, como pensar a arquitetura pedagógica para comportar tais diferenças e fomentar uma forma de pensar a resolução de problemas nas mais diversas áreas do conhecimento.

O debate deixa claro que precisamos de novos trabalhos, novas pesquisas voltadas a essa temática, parâmetros de mensuração de dados coletados com as atividades desenvolvidas a partir dos parâmetros do Pensamento Computacional inclusivo e, sobretudo, medidas objetivas de maior aproveitamento dos estudos desses alunos com deficiência e altas habilidades ou superdotação.

\section{Referências}

American Psychiatric Association. (2013). Diagnostic and Statistical Manual of Mental Disorders, Fifth Edition (DSM-V). Arlington, VA: American Psychiatric Association.

Andrade, D., Carvalho, T., Silveira, J., Cavalheiro, S., Foss, L., Fleischmann, A. M., ... \& Reiser, R. (2013). Proposta de atividades para o desenvolvimento do pensamento computacional no ensino fundamental. In Anais do Workshop de Informática na Escola (Vol. 19, No. 1, p. 169).

Araújo, C. A. A. (2006). Bibliometria: evolução histórica e questões atuais. Em questão, 12.1: 11-32.

Barab, S. A., \& Roth, W. (2006) Curriculum-based ecosystems: Supporting knowing from an ecological perspective. Educational Researcher, 35 (5), 3-13

Barbosa, F. D. A. C., \& China, A. P. Z. (2017). Aulas de informática na grade curricular da educação infantil e ensino fundamental: importância e benefícios para a formação integral. Revista Interface Tecnológica, 14(1), 9-20.

Barcelos, T., Muñoz, R., Acevedo, R. V., \& Silveira, I. F. (2015). Relações entre o pensamento computacional e a matemática: uma revisão sistemática da literatura. In Anais dos Workshops do Congresso Brasileiro de Informática na Educação (Vol. 4, No. 1, p. 1369).

Barcelos, T. S., \& Silveira, I. F. (2012). Pensamento computacional e educação matemática: Relações para o ensino de computação na educação básica. In: 2012, Curitiba. XX Workshop sobre Educação em Computação, Curitiba. Anais do XXXII CSBC. Curitiba: UFPR, p. 23.

Barr, V. \& Stephenson, C. (2011). Bringing computational thinking to K-12. ACM Inroads, New York, NY, v. 2, n. 1, p. 48-54.

Barwaldt, R., Santarosa, L. M. C., \& Passerino, L. M. (2008). Uma ferramenta de autoria síncrona acessível para cegos: um estudo de caso no PROINESP. RENOTE: Revista Novas Tecnologias na Educação, Porto Alegre, v. 6, n. 2, dez.

Bauer, C. M., Hirsch, G. V., Zajac, L., Koo, B. B., Collignon, O., \& Merabet, L. B. (2017). Multimodal MR-imaging reveals large-scale structural and functional connectivity changes in profound early blindness. PLoS One, 12(3), e0173064.

Bell, T., Witten, I. H., Fellows, M., Adams, R., \& McKenzie, J. (2011). Ensinando Ciência da Computação sem o uso do computador. Computer Science Unplugged ORG.

Beytien, A. (2011). Autismevery day. Arlington: Future Horizons Inc.

Brasil. (2017). Base Nacional Comum Curricular: Matemática. Brasília, p. 268-319.

Brasil. (2020). Decreto $n^{o}$ 10.502, de 30 de setembro de 2020. Institui a Política Nacional de Educação Especial: Equitativa, Inclusiva e com Aprendizado ao Longo da Vida. Diário Oficial [da] República Federativa do Brasil, Brasília.

Brasil. (2021b). Instituto Nacional de Estudos e Pesquisas Educacionais Anísio Teixeira. Censo da Educação Básica 2020: notas estatísticas. Brasília, DF: INEP.

Brasil. (2015). Lei $n^{o}$ 13.146, de 6 de julho de 2015. Institui a Lei Brasileira de Inclusão da Pessoa com Deficiência (Estatuto da Pessoa com Deficiência). Diário Oficial [da] República Federativa do Brasil, Brasília.

Brasil. (2021 a) Lei $n^{o}$ 14.172, DE 10 DE JUNHO DE 2021. Dispõe sobre a garantia de acesso à internet, com fins educacionais. DF.

Brasil. (2018). Ministério da Educação. Base Nacional Comum Curricular: Educação é a base. Brasília: MEC. 
Brasil. (2008). Ministério da Educação. Secretaria da Educação Especial. Política Nacional de Educação Especial na Perspectiva da Educação Inclusiva. MEC/SEESP, Brasília.

Brasil. (2021c). Supremo Tribunal Federal (STF). Política nacional de educação especial [recurso eletrônico]: equitativa, inclusiva e com aprendizado ao longo da vida - PNEE: (Decreto $\mathrm{n}^{\circ}$ 10.502, de 30 de setembro de 2020) : bibliografia, legislação e jurisprudência temática / Supremo Tribunal Federal. Brasília: STF, Secretaria de Altos Estudos, Pesquisas e Gestão da Informação.

Brackmann, C. P. (2017). Desenvolvimento do pensamento computacional através de atividades desplugadas na educação básica.

Comin, S., \& Silva, D. (2020). Neuróbica para desenvolvimento de alunos com deficiência intelectual. Faculdade Sant'Ana Em Revista, 4(1), p. 109-122. https://www.iessa.edu.br/revista/index.php/fsr/article/view/1236.

CSTA (2013). Bugs in the System: Computer science teacher certification in the U.S. New York, NY: Computer Science Teachers Association.

Dantas, S. A. L. (2019). Robótica de baixo custo como objeto de aprendizagem para estudantes com altas habilidades ou superdotação.

Fava, R. (2016). Educação para o século XXI: a era do indivíduo digital. São Paulo: Saraiva.

França, R. S., \& Tedesco, P. C. A. R. (2015). Desafios e oportunidades ao ensino do pensamento computacional na educação básica no Brasil. In: Anais do Congresso Brasileiro de Informática na Educação, pp. 4-5, SBC, Maceió, Brasil.

Giroto, C. R. M., Poker, R. B., \& Omote, S. (ed.). (2012). As tecnologias nas práticas pedagógicas inclusivas. Editora Oficina Universitária.

Glat, R. (2007). Educação Inclusiva: Cultura e Cotidiano Escolar. 7letras.

Gomes, D. E. L. (2021). Educação especial e inclusiva em escolas públicas do município de Tomé-Açu: acompanhamento de alunos com TEA.

Guarda, G. F., \& Pinto, S. C. C. S. (2020). Dimensões do Pensamento Computacional: conceitos, práticas e novas perspectivas. In Anais do XXXI Simpósio Brasileiro de Informática na Educação (pp. 1463-1472). SBC.

Guarda, G. F., \& Pinto, S. C. C. S. (2021). O uso dos jogos digitais educacionais no processo no ensino-aprendizagem com ênfase nas habilidades do pensamento computacional. Revista Brasileira de Pós-Graduação, 17(37), 1-35.

Hadwen-bennett, A., Sentance, S., \& Morrison, C. (2018). Making Programming Accessible to Learners with Visual Impairments: A Literature Review. International Journal of Computer Science Education in Schools, v. 2, n. 2, p. 3-13.

Jackson, N. (2013). Lifewide learning, education \& personal development. The concept of.

Joia, M., \& Braz, R. M. (2021). Relato de Experiência sobre a utilização de Materiais Adaptados para Alunos com Tea de acordo com Nivelamento Cognitivo. I Workshop Ibero Americano de Educação Inclusiva. https://bit.ly/2TJjsH8.

Katz, L. C., \& Rubin, M. (2000). Mantenha o Seu Cérebro Vivo: exercícios neuróbicos para ajudar a prevenir a perda de memória e aumentar a capacidade mental. Tradução de Alfredo Barcellos Pinheiro Lemos. Rio de Janeiro: Sextante.

Leite, E., \& Braz, R. M. (2021). Relato de Experiência sobre a construção de um novo olhar: formação inicial/continuada em práticas pedagógicas inclusivas na perspectiva do Desenho Universal para Aprendizagem (DUA). I Worhkshop Ibero Americano de Educação Inclusiva. https://bit.ly/2TJjsH8.

Lima, C. B. (2012). Perturbações do espectro do autismo: Manual prático de intervenção. Lisboa: Lidel - Edições técnicas.

Leon, V. F., \& Fonseca, M. M. (2013). Contribuições do Ensino Estruturado na Educação de Crianças e Adolescentes com Trantorno do Espectro do Autismo. Autismo, educação e transdisciplinaridade. Campinas, SP: Papirus.

Lu, J. J., \& Fletcher, G. H. L. (2009). Thinking about computational thinking. ACM SIGCSE Bulletin, New York, NY, vol. 41, no. 1, p. 260-264, Mar.

Mašić, V., Šečić, A., Bobić, T. T., \& Femec, L. (2020). Neuroplasticity and Braille reading. Acta Clin Croat, 59(1):147-153.

Monteiro, E. M. M. (2017). Avaliação e treino cognitivo de crianças com incapacidade intelectual.

Morrison, C., Villar, N., Thieme, A., Ashktorab, Z., Taysom, E., Salandin, O., \& Zhang, H. (2020). Torino: A tangible programming language inclusive of children with visual disabilities. Human-Computer Interaction, 35(3), 191-239.

Nunes, S. S., \& Lomônaco, J. F. B. (2008). Desenvolvimento de conceitos em cegos congênitos: caminhos de aquisição do conhecimento. Revista Semestral da Associação Brasileira de Psicologia Escolar e Educacional, Campinas, v. 12, n. 1, p. 119-138.

Papert, S. (1980). Mindstorms: Children, computers, and powerful ideas. New York, NY: Basic Books.

Piaget, J. (1998). Para onde vai a educação? Tradução de Ivete Braga, 14ª edição. Rio de Janeiro: José Olímpio.

Pinto, S. C. C. S; \& Nascimento, G. S. R. (2018). O pensamento computacional e a nova sociedade. In: José Armando Valente, Fernanda Maria Pereira Freire e Flávia Linhalis Arantes. (Org.). Tecnologia e educação: passado, presente e o que está por vir.1ed. Campinas: NIED/UNICAMP, v. 1, p. $302-322$.

Ramos, H. D. A. (2014). Pensamento Computacional na Educação Básica: uma proposta de aplicação pedagógica para alunos do quinto ano do Ensino Fundamental do Distrito Federal. https://bdm.unb.br/handle/10483/7720

Rezende, S. M., \& Pinto, S. C. C. S. (2021). O uso de tecnologias digitais em objetos de aprendizagem da matemática para estudantes com deficiência visual: um levantamento de teses e dissertações brasileiras. Caminhos da educação matemática em revista (online)/IFS, v. 11, n. 3. 
Research, Society and Development, v. 10, n. 14, e400101421789, 2021

(CC BY 4.0) | ISSN 2525-3409 | DOI: http://dx.doi.org/10.33448/rsd-v10i14.21789

Renzulli, J. S. (1986). The three-ring conception of giftedness: a developmental model for creative productivity. In: R. J. Sternberg \& J. E. Davis (Eds.) Conceptions of giftedness (pp. 53-92). New York: Cambridge University Press.

Riazy, S., Weller, S., \& Simbeck, K. (2020). Evaluation of Low-threshold Programming Learning Environments for the Blind and Partially Sighted: In: Proceedings of the 12th International Conference on Computer Supported Education. Prague, Czech Republic: SCITEPRESS - Science and Technology Publications, p. 366-373.

Santin, S., \& Simmons, J. (1996). Problemas das crianças portadoras de deficiência visual congênita na construção da realidade. Trad. de Ilza Viega. Revista Benjamin Constant, edição 02, p. 7-11.

Senid. (2021). Seminário Internacional de Inclusão Digital. https://www.upf.br/senid.

Stainback, W., \& Stainback, S. (1999). Fundamentos do ensino inclusivo. Inclusão: um guia para educadores, p. 21-34.

Unesco. (2020). Relatório de monitoramento global da educação - resumo, 2020: In clusão e educação: todos, sem exceção. https://bit.ly/3xQOtIs.

Who - World Health Organization. (2021). World report on hearing Geneva. 2021. ISBN 9789240020481. https://apps. who.int/iris/handle/10665/339913

Wing, J. M. (2020). Computational Thinking. Communications of the ACM, [S.I.], v. 49, n. 3, p. 33-35, mar. 2006. https://www.cs.cmu.edu/ 15110s13/Wing06-ct.pdf.

Wing, J. M. (2010). Computational Thinking: What and Why? Unpublished Manuscript, Pittsburgh, PA: Computer Science Department, Carnegie Mellon University, 2010. 